\title{
Estratégias de acessibilidade e recursos didáticos utilizados em softwares educacionais para surdos: uma revisão de literatura
}

\author{
Hellen S. Luz ${ }^{1}$, Magaly L. C. Campos ${ }^{1}$, George França ${ }^{1}$, David Prata Nadler ${ }^{1}$ \\ ${ }^{1}$ Programa de Pós-Graduação em Modelagem Computacional de Sistemas - \\ Universidade Federal do Tocantins (UFT) \\ Palmas - TO - Brasil \\ \{hellen.luz,magaly\}@ifto.edu.br, george_franca@yahoo.com, \\ ddnprata@gmail.com
}

\begin{abstract}
This article reviews studies with the objective of knowing and analyzing the strategies of some productions about the use of software aiming at the teaching and learning of deaf students. For this purpose, we searched the databases of national journals and later the analysis of the results was performed. The research has shown that software has helped the deaf community, especially with tools for learning the Portuguese language and LIBRAS (Linguagem Brasileira de Sinais - Brazilian Signal Language), but with efforts in the development of virtual environments, in the development of games, translators and dictionaries.
\end{abstract}

Resumo. $O$ presente artigo faz uma revisão de estudos com o objetivo de conhecer $e$ analisar as estratégias usadas para acessibilidade e para mediação da aprendizagem em softwares desenvolvidos ou utilizados para o ensino e aprendizagem de alunos surdos. Com esse intuito, efetuou-se buscas na base de dados de periódicos nacionais e, posteriormente, realizou-se análise dos resultados encontrados. A investigação mostrou que o uso de dicionários e tradutores, jogos e exercícios individuais são as principais estratégias utilizadas em softwares para a mediação da aprendizagem do surdo. Em relação à acessibilidade, o uso massivo de imagens como ícones $e$ a utilização de textos curtos são os principais recursos.

\section{Introdução}

Segundo Censo Escolar realizado pelo Instituto Nacional de Estudos e Pesquisas Anísio Teixeira - INEP, no ano 2017, o número de matrículas na Educação especial, em classes exclusivas e/ou classes comuns, no Ensino Regular, Especial e/ou Educação de Jovens e Adultos (EJA), era de 1.066.446. Do total de matriculados, 25.507 apresentavam surdez, 39.075 tinham deficiência auditiva e 420 possuíam surdocegueira.

A legislação Brasileira assegura não só a inclusão dos alunos com deficiência, mas os recursos físicos, humanos, financeiros, currículos, métodos, técnicas, recursos educativos e organização específicos indispensáveis para efetivá-la. Porém, percebe-se que a inclusão dos alunos em classes regulares não tem sido acompanhada de reformas necessárias, em especial, as pedagógicas. Faz-se necessário investimento em tecnologias que favoreçam não somente a inclusão dos alunos surdos nas classes comuns, mas também sua inclusão no processo educacional.

Tendo em vista os estudos sobre o desenvolvimento do surdo e a sua língua na modalidade visual-espacial, o uso de tecnologias digitais da informação e comunicação, dentre elas o computador, pode favorecer a proposição de alternativas mais condizentes com as demandas atuais dos alunos surdos, uma vez que estas vêm se constituindo em valiosas ferramentas de apoio na superação das desigualdades e na inclusão social. 
VII Congresso Brasileiro de Informática na Educação (CBIE 2018)

Anais do XXIV Workshop de Informática na Escola (WIE 2018)

Entretanto, falar de computador na educação implica pensar e falar de software, e ainda, conforme nos adverte Valente,

"mais do que discutir qual o software ideal, devemos indagar o que se
considera como aprendizagem, que condições a favorecem e como se pode
criá-las. A partir daí, sim, pensar quais software podem ser usados e em quais
condiçóes passam a ser mais uma situação na qual pode-se repensar práticas
pedagógicas e conceitos sobre aprendizagem." [Petry, 1997, apud Valente,
1999, p. 67]

Tendo como pressuposto a educação libertadora, em que "[...] ensinar não é transferir conhecimento, mas criar as possibilidades para sua produção ou construção" [Freire, 1996, p. 21] e o computador como ferramenta de aprendizagem, faz-se necessário repensar o papel do professor no processo. Este deixa de ser o eixo central, o único detentor do conhecimento e, conforme Toschi,

"passa a ser o mediador do conhecimento instigando os alunos no que se refere à construção do saber, dando abertura para que possam construir seus próprios conhecimentos, valendo-se das mídias como meio para aprendizagem(....) E como meio, os educadores, deverão fazer uso das mídias, para mediar a transformação das informações em conhecimento no processo ensino aprendizagem." [Toshi, 2010, p. 149]

Porém, nem todas as pessoas têm possibilidade de acessar os recursos de hardware ou software que o mundo digital oferece devido a limitações, no caso dos surdos, auditivas. Para potencializar as capacidades destas pessoas, torna-se indispensável o uso de tecnologias que, conforme Bersch (2006), pode ser considerada assistiva quando percebe-se que, sem este recurso tecnológico, a participação ativa do aluno no desafio de aprendizagem seria restrito ou inexistente.

Almeida e Valente (2001) ressaltam a importância da análise das potencialidades dos softwares, ferramentas e interfaces nos processos de ensino e aprendizagem, uma vez que esta permite entender o papel das tecnologias digitais de informação e comunicação nos processos de ensino e aprendizagem

Nesse sentido, ao se pensar na utilização ou na construção de um software para apoiar o processo de ensino e aprendizagem de alunos surdos, há que se considerar dois aspectos fundamentais: a acessibilidade e as estratégias disponibilizadas pelo ambiente digital para a mediação da aprendizagem.

Diante do exposto, uma revisão de literatura foi realizada com o objetivo de identificar e analisar na produção científica referente a software para a educação de surdos, as estratégias para a acessibilidade e para a mediação pedagógica utilizadas.

Este trabalho está organizado da seguinte forma: A primeira seção contextualiza o trabalho; a segunda aborda o método utilizado na pesquisa; na terceira são apresentados os resultados e na quarta são apresentadas algumas reflexões e trabalhos futuros sobre o tema.

\section{Metodologia}

Em busca de identificar as estratégias de acessibilidade e mediação da aprendizagem em software para o apoio ao ensino e aprendizagem de surdos um protocolo baseado no método de Revisão Sistemática da Literatura (RSL), proposta por Kitchenham e Charters (2007), foi desenvolvido.

Dessa forma, estudos que apresentaram softwares voltados para o ensino ou aprendizagem de surdos foram recuperados para, posteriormente, responder as seguintes questões:

[Q1] Quais tipos de recursos de acessibilidade são utilizados nos softwares para 
VII Congresso Brasileiro de Informática na Educação (CBIE 2018)

Anais do XXIV Workshop de Informática na Escola (WIE 2018)

possibilitar a inclusão dos surdos?

[Q2] Quais recursos pedagógicos são utilizados nos softwares?

Para a recuperação dos estudos, uma string de busca foi gerada a partir da combinação dos termos "software", "ensino", "aprendizagem", "surdo" com seus sinônimos usando os operadores lógicos OR e AND. As bases de busca utilizadas foram: Simpósio Brasileiro de Informática na Educação - SBIE, Workshop de Informática na Escola - WIE, Workshops do Congresso Brasileiro de Informática na Educação - WCBIE, Jornada de Atualização de Informática na Educação - JAIE; Workshop de Desafios da Computação Aplicada à Educação - WDCAE, Revista Brasileira de Informática na Educação - RBIE, Revista Novas Tecnologias na Educação - Renote, Revista Científica Internacional - InterSciencePlace.

Antes da realização do mapeamento junto aos periódicos foram elaborados, com o objetivo de manter a organização, clareza e a atualidade da pesquisa, critérios de exclusão e uma planilha para o extrato das análises dos artigos.

Foram excluídos: 1) Estudos realizados antes do ano 2007. 2) Revisões e mapeamentos sistemáticos de literatura. 3) Estudos que não respondam nenhuma das questões norteadoras. 4) Estudos duplicados ou redundantes (para estudos de mesma autoria ou relacionados à mesma solução, apenas o mais recente e/ou o mais completo foi incluído, a menos que tivesse informação complementar). 5) Estudos que não apresentam uma proposta de software.

Após a realização da busca e seleção dos estudos, a extração dos dados aconteceu em duas fases: Fase 01 - Análise dos Títulos e Resumos, Fase 02 - Análise completa dos artigos.

A fase 01 teve por objetivo verificar se os artigos auxiliariam a responder a questão da pesquisa. Nela, foi realizada a leitura individual por cada uma das pesquisadoras e a identificação de inclusão ou exclusão do estudo a partir dos critérios estabelecidos. Nessa fase foram incluídos 24 artigos, 10 deles tiveram orientações divergentes quanto à inclusão ou exclusão no estudo e 69 artigos foram excluídos, de acordo com os critérios estabelecidos.

$\mathrm{Na}$ fase 02 da análise foram realizadas as leituras integrais dos 24 artigos incluídos na $1^{\mathrm{a}}$ fase e dos 10 com orientações divergentes, resultando na inclusão de 3 , totalizando 27 artigos. Dessa forma, dos 103 artigos, 74\% foram excluídos, 76 artigos, sendo $15 \%$ pelo critério $1,4 \%$ pelo critério $2,51 \%$ pelo critério $3,3 \%$ pelo critério 4 e $1 \%$ pelo critério 5 .

Durante a leitura integral dos artigos, os softwares apresentados foram classificados de acordo com os estudos, seguindo a estrutura: a) Nome do Software b) Tipo de Software c) Base de Dados d) Ano e) Título do artigo f) Autores g) Estratégias de Acessibilidade h) Estratégias de Mediação.

\section{Resultados e Discussões}

A análise dos 27 artigos selecionados para a revisão de estudos possibilitou identificar 29 softwares, apresentados pelos autores elencados na tabela 1. Entre eles, foram encontrados as seguintes soluções: Jogos, Ambientes de Aprendizagem, Ferramentas de Apoio à Aprendizagem, Tradutores, Dicionários, Teclados para a digitação em língua de sinais e Objetos de Aprendizagem. A classificação apresentada seguiu a denominação atribuída pelos autores das pesquisas. 
VII Congresso Brasileiro de Informática na Educação (CBIE 2018)

Anais do XXIV Workshop de Informática na Escola (WIE 2018)

Tabela 1. Softwares analisados.

\begin{tabular}{|c|c|c|c|c|c|}
\hline ID & TIPO DE SOFTWARE & NOME & ID & $\begin{array}{c}\text { TIPO DE } \\
\text { SOFTWARE }\end{array}$ & NOME \\
\hline S01 & $\begin{array}{l}\text { Ambiente de } \\
\text { Aprendizagem }\end{array}$ & Eduquito & S16 & \multirow{3}{*}{ Tradutores } & Tradutor HandTalk \\
\hline S02 & $\begin{array}{l}\text { Ambiente Virtual de } \\
\text { aprendizagem }\end{array}$ & $\begin{array}{l}\text { Agente Pedagógico } \\
\text { Clóvis }\end{array}$ & S17 & & Tradutor ProDeaf \\
\hline S03 & $\begin{array}{l}\text { Fórum de discussão que } \\
\text { utiliza a SWService }\end{array}$ & Sign WebForum & S18 & & Tradutor Rybená \\
\hline S04 & $\begin{array}{l}\text { Ferramenta de apoio à } \\
\text { aprendizagem }\end{array}$ & \begin{tabular}{|l} 
Sistema Tutor \\
Inteligente
\end{tabular} & S16 & \multirow{2}{*}{ Tradutores } & Tradutor HandTalk \\
\hline S05 & $\begin{array}{l}\text { Teclado Virtual para a } \\
\text { escrita da LIBRAS }\end{array}$ & Teclado Virtual & S17 & & Tradutor ProDeaf \\
\hline S06 & $\begin{array}{l}\text { Ferramenta de apoio à } \\
\text { aprendizagem }\end{array}$ & SAEPS & S19 & $\begin{array}{l}\text { Ambiente Digital } \\
\text { de Aprendizagem }\end{array}$ & $\begin{array}{l}\text { MVLIBRAS - } \\
\text { Plataforma CAP }\end{array}$ \\
\hline S07 & Jogos digitais & $\begin{array}{l}\text { Jogos digitais a } \\
\text { partir da mediação } \\
\text { do software Scratch }\end{array}$ & S20 & $\begin{array}{l}\text { Ferramenta de } \\
\text { apoio à } \\
\text { aprendizagem }\end{array}$ & $\begin{array}{l}\text { Ferramenta de } \\
\text { reforço de } \\
\text { vocabulário } \\
\end{array}$ \\
\hline S08 & $\begin{array}{l}\text { Ferramenta de apoio à } \\
\text { aprendizagem. }\end{array}$ & DeafWord & $\mathbf{S 2 1}$ & Jogo & \begin{tabular}{|l} 
Lectoescritura con \\
Fitzgerald
\end{tabular} \\
\hline S09 & $\begin{array}{l}\text { Ferramenta de apoio à } \\
\text { aprendizagem }\end{array}$ & MCHQ-Alfa & $\mathbf{S 2 2}$ & Jogo & VirtualSign \\
\hline S10 & Glossário & Glossário & $\mathbf{S 2 3}$ & $\begin{array}{l}\text { Objeto de } \\
\text { Aprendizagem }\end{array}$ & Lepê \\
\hline S11 & Jogo da memória & $\begin{array}{l}\text { JMLP - Jogo da } \\
\text { Memória Libras e } \\
\text { Português }\end{array}$ & S24 & Jogo & LibrasZap \\
\hline S12 & $\begin{array}{l}\text { Objeto de aprendizagem } \\
\text { acessível }\end{array}$ & VISUAL JO2 & S25 & Tradutor & E-Sinais \\
\hline $\mathbf{S 1 3}$ & \multirow[b]{2}{*}{ Jogos } & Jogo Libr@snet & S26 & Jogo & Serious Libras \\
\hline S14 & & Jogo Multi-Trilhas & S27 & $\begin{array}{l}\text { Ambiente de } \\
\text { Aprendizagem }\end{array}$ & GeoLIBRAS \\
\hline \multirow[t]{2}{*}{ S15 } & Dicionário & $\begin{array}{l}\text { Dicionário da } \\
\text { Língua Brasileira } \\
\text { de Sinais } \\
\end{array}$ & $\mathbf{S 2 8}$ & Dicionário & $\begin{array}{l}\text { Dicionário de } \\
\text { Termos da } \\
\text { Computação } \\
\end{array}$ \\
\hline & & & S29 & Dicionário & LibrasTI \\
\hline
\end{tabular}

\subsection{Estratégias de Acessibilidade de Interface}

A análise nos proporcionou identificar 11 diferentes estratégias de acessibilidade de interface para surdos, utilizadas no desenvolvimento dos softwares educativos encontrados.

Dentre essas estratégias, identificou-se que 18 softwares fizeram o uso massivo de cores e ícones e/ou imagens como metáforas do mundo real para auxiliar o surdo no seu uso. Em geral, essa estratégia foi utilizada em combinação com textos curtos associados às imagens.

$\mathrm{Na}$ sequência, três estratégias que podem ser consideradas específicas para os surdos foram identificadas em 12 softwares: uso de vídeos em LIBRAS como apoio na apresentação inicial da ferramenta (5 softwares), a utilização da configuração de mãos no processo de tradução de palavras do português para a LIBRAS em locais específicos dentro dos ambientes (4 softwares) e a utilização de avatares no apoio ao processo de interação (4 softwares). Entre esses softwares, um ambiente virtual de aprendizagem se apoia tanto no uso de um vídeo em LIBRAS para a apresentação da ferramenta quanto 
VII Congresso Brasileiro de Informática na Educação (CBIE 2018)

Anais do XXIV Workshop de Informática na Escola (WIE 2018)

no uso de um avatar no apoio à interação do aluno com o ambiente. Nessa ferramenta, o avatar possui o diferencial de que ele não realiza sinais em LIBRAS, mas utiliza expressões que demonstram diversos tipos de emoção para apoiar o uso.

Tabela 2. Estratégias de Acessibilidade.

\begin{tabular}{l|l|}
\hline \multicolumn{2}{|c|}{ ESTRATÉGIAS DE ACESSIBILIDADE DE INTERFACE } \\
\hline \multicolumn{1}{|c|}{ ESTRATÉGIAS } & \multicolumn{1}{c}{ SOFTWARES } \\
\hline Padrões W3C, E-MAG & S01, S29 \\
\hline $\begin{array}{l}\text { Vídeo em Libras para apresentação do } \\
\text { ambiente }\end{array}$ & S01, S02, S05, S14, S23 \\
\hline $\begin{array}{l}\text { Navegação através de animações/vídeos } \\
\text { em Libras }\end{array}$ & S08, S12, S19, S23 \\
\hline $\begin{array}{l}\text { Exploração de imagens - ícones, metafóras, } \\
\text { figuras e cores }\end{array}$ & $\begin{array}{l}\text { S01, S04, S05, S06, S07, S08, S09, S12, S21, } \\
\text { S14, S19, S20, S11, S14, S23, S25, S27, S29 }\end{array}$ \\
\hline $\begin{array}{l}\text { Utilização de textos curtos } \\
\text { S01, S07, S08, S19, S23, S24, S25, S26, S27, } \\
\text { S28, S29 }\end{array}$ \\
\hline $\begin{array}{l}\text { Uso da Interface Natural } \\
\text { Uso de interface Tangível }\end{array}$ & S06, S22 \\
\hline $\begin{array}{l}\text { Interface sinalizada em libras configuração } \\
\text { de mãos }\end{array}$ & S03, S09, S10, S15 \\
\hline $\begin{array}{l}\text { Interface sinalizada em libras com escrita } \\
\text { de sinais }\end{array}$ & S03 \\
\hline Avatares & S02, S16, S17, S18 \\
\hline Não foi possível avaliar & S13 \\
\hline
\end{tabular}

\subsection{Estratégias para a mediação da aprendizagem do surdo em ambientes educativos}

Ainda foi possível identificar diversas estratégias propostas pelos estudos e implementadas nos softwares para a mediação da aprendizagem de indivíduos surdos. Considerando que grande parte das iniciativas aqui discutidas se propõe a ensinar o português como uma segunda língua para o surdo, estratégias como a consulta a termos e frases com o uso de dicionários e tradutores (13 softwares) bem como a produção de textos (2 softwares), clássicas no ensino de ouvintes, também aparecem aqui em um conjunto considerável de softwares.

Observa-se ainda um grande número de propostas que apostam na ludicidade dos jogos como estratégia de engajamento e mediação da aprendizagem (9 softwares). O jogos, em sua maioria, se propõe a ensinar o português e a LIBRAS ao surdo, sendo possível identificar ambientes que oferecem o recurso de gamificação aos professores.

Um recurso clássico e que se apresenta como uma das estratégias mais utilizadas é a realização de exercícios individuais (8 softwares). Nelas, em geral, os surdos são convidados a desenvolver atividades em que deverão relacionar sinais em LIBRAS a imagens, aprendendo através do método da tentativa e erro. 
VII Congresso Brasileiro de Informática na Educação (CBIE 2018)

Anais do XXIV Workshop de Informática na Escola (WIE 2018)

Tabela 3. Estratégias de Mediação.

\begin{tabular}{|l|l|}
\hline \multicolumn{2}{|c|}{ ESTRATÉGIAS DE MEDIAÇÃO DA APRENDIZAGEM } \\
\hline \begin{tabular}{l}
\multicolumn{1}{c|}{ ESTRATÉGIAS* } \\
Desenvolvimento de atividades \\
colaborativas
\end{tabular} & S01, S10, S19 \\
\hline Exercícios individuais & $\mathrm{S} 02, \mathrm{~S} 06, \mathrm{~S} 20, \mathrm{~S} 04, \mathrm{~S} 11, \mathrm{~S} 12, \mathrm{~S} 27, \mathrm{~S} 23$ \\
\hline Jogos & $\mathrm{S} 22, \mathrm{~S} 06, \mathrm{~S} 21, \mathrm{~S} 11, \mathrm{~S} 13, \mathrm{~S} 14, \mathrm{~S} 24, \mathrm{~S} 26, \mathrm{~S} 27$ \\
\hline Produção de Textos & $\mathrm{S} 08, \mathrm{~S} 05$ \\
\hline Construção de mapas conceituais & $\mathrm{S} 09$ \\
\hline Criação de Jogos & $\mathrm{S} 07$ \\
\hline Apresentação de conteúdos & $\mathrm{S} 04, \mathrm{~S} 12, \mathrm{~S} 27$ \\
\hline $\begin{array}{l}\text { Uso do português escrito na estrutura } \\
\text { gramatical da Libras }\end{array}$ & $\mathrm{S} 15, \mathrm{~S} 27$ \\
\hline Fórum de discussão & $\mathrm{S} 01, \mathrm{~S} 03$ \\
\hline Consulta de termos e frases & $\begin{array}{l}\text { S06, S08, S10, S15, S16, S17, S18, S19, S20, } \\
\text { S21, S22, S28, S29 }\end{array}$ \\
\hline & \\
\hline * Existem softwares que apresentam mais de uma estratégia \\
\hline
\end{tabular}

\section{Considerações Finais}

O objetivo deste estudo foi identificar as estratégias de acessibilidade e mediação pedagógica utilizadas em softwares no apoio ao processo de ensino e aprendizagem de estudantes surdos. Para isso, uma revisão de literatura foi realizada.

Observou-se um uso massivo da exploração de imagens na forma de metáforas ou ícones como estratégia de acessibilidade. Seu uso justifica-se pela visualidade do sujeito surdo que, sem a compreensão da língua portuguesa, encontra apoio para a navegação nos ambientes através dos recursos visuais.

No uso dessas estratégias para a construção de ferramentas educacionais ou para o apoio ao processo de aprendizagem de alunos, deve-se lembrar que as imagens utilizadas precisam fazer parte do contexto do público alvo, para que ele possa reconhecer a ação requerida na observação da imagem. A conjugação de seu uso com recursos como textos curtos e glosas no formato de dicas pode auxiliar o surdo, cuja imagem representada como ícone ou metáfora não lhe é familiar. Além dessa estratégia, a utilização de padrões utilizados em outros softwares familiares como as redes sociais também pode colaborar.

Em relação à mediação pedagógica, observou-se o uso da consulta a termos, os jogos e a realização de exercícios individuais como as principais estratégias. Considerando que a LIBRAS é uma língua em rápida evolução e que novos sinais são criados para representar conceitos antes inexistentes na língua em diversas áreas do conhecimento, o registro e o compartilhamento rápido de informações é fundamental para que os professores trabalhem novos conteúdos.

Por fim, acredita-se que a inclusão dos surdos nos espaços de aprendizagem devem contemplar, além da excelência de materiais e profissionais, o processo de troca, de socialização e o profundo respeito por sua língua e cultura. Esse respeito deve transparecer também na utilização de estratégias de acessibilidade coerentes e em recursos pedagógicos que os favoreçam. 
VII Congresso Brasileiro de Informática na Educação (CBIE 2018)

Anais do XXIV Workshop de Informática na Escola (WIE 2018)

\section{Referências}

Almeida, M. E. B., Valente, J. A. (2011) "Tecnologias e Currículo: trajetórias convergentes ou divergentes?", In: São Paulo: Paulus.

Araújo, S., Silveira, D. and Matos, P. (2017) "Avaliação do Software Educacional eSinais no Ensino-Aprendizagem da Língua Portuguesa Escrita e da L I B R A S”, In: XXIII Workshop de Informática na Escola, page 323. Disponível em: <http://www.br-ie.org/pub/index.php/wie/article/view/7250>. Acesso em: 15 jun.2018.

Barth, C., Santarosa, L. M. C. And Schneider, F. C. et al. (2009) "Construção da Leitura/Escrita em Língua de Sinais de Crianças Surdas em Ambientes Digitais”, In: Novas Tecnologias na Educação, V. 7, n.N. 03.

Bersch, R. (2006) “Tecnologia assistiva e educação inclusiva”, In: Ensaios Pedagógicos, Brasília: SEESP/MEC, pages 89-94.

Brasil, Subsecretaria Nacional de Promoção dos Direitos da Pessoa com Deficiência. (2009) Comitê de Ajudas Técnicas Tecnologia Assistiva. - Brasília : CORDE, 138 p.

Brito, B. P. and Ferreira, B. J (2015) "Proposta de uma Ferramenta de Reforço de Vocabulário na Educação de Surdos", In: Novas Tecnologias na Educação, v. 13, n. 1. (CINTED-UFRGS).

Cano, S., Arteaga, J. M., Collazos, C. and et al. (2015) "Aplicación Móvil para el aprendizaje de la lectoescritura con Fitzgerald para niños con discapacidad auditiva", In: IV Congresso Brasileiro de Informática na Educação, p. 240. Disponível em: <http://br- ie.org/pub/index.php/wcbie/article/view/5983>. Acesso em: 15 jun. 2018.

Colling, J. P. and Boscarioli, C. (2014) Avaliação de tecnologias de tradução portuguêslibras visando o uso no ensino de crianças surdas, In: CINTED - Novas tecnologias na educação. v. 12, p. 10. UFRGS.

Cruz, S. M. S. D., Calé, F., Paim, L. and et al. (2017) "Uma ferramenta para auxiliar o ensino da Tecnologia da Informação para surdos", In: VI Congresso Brasileiro de Informática na Educação, p. 244. Disponível em: <http://www.brie.org/pub/index.php/wcbie/article/view/7387>. Acesso em: 15 jun. 2018.

D'ávila, C. (2012) "Interdisciplinaridade e mediação: desafios no planejamento e na prática pedagógica da educação superior", In: Conhecimento \& Diversidade, v. 3, n. 6, p. 58-70.

Escudeiro, P. (2015) “Jogos Sérios para Língua Gestual Portuguesa”, In: IV Congresso Brasileiro de Informática na Educação, p. 170. Disponível em: <http://brie.org/pub/index.php/wcbie/article/view/5963>. Acesso em: 15 jun. 2018.

Freire, P. (1996) "Pedagogia da autonomia: saberes necessários à prática docente", In: São Paulo: Paz e Terra, p. 90.

Granada, R., Cesário, V., Domingues, D. and et al. (2017) "Dicionário de termos de computação como facilitador no ensino de programação para surdos", In: VI Congresso Brasileiro de Informática na Educação, p. 1049. Disponível em: $<$ http://www.br-ie.org/pub/index.php/wcbie/article/view/7494>. Acesso em: 15 jun. 2018 .

INEP, Instituto Nacional de Estudos e Pesquisas Anisio Teixera. (2017) "Sinopse Estatística da Educação Básica 2017”, In: Brasília: Inep, 2018. Disponível em:< http://inep.gov.br/sinopses-estatisticas-da-educacao-basica> Acesso em 10 maio.2018 
VII Congresso Brasileiro de Informática na Educação (CBIE 2018)

Anais do XXIV Workshop de Informática na Escola (WIE 2018)

Kitchenham, B. and Charters, S. (2007) "Guidelines for performing systematic literature reviews in software engineering". Keele University and Durham University Joint Report, Tech. Rep. EBSE 2007-001, 2007.

Moura, E. R. S. and Oliveira, E. H. T. (2014) "Uma ferramenta colaborativa móvel para apoiar o processo de ensino-aprendizagem da LIBRAS e do Português para surdos", In: III Congresso Brasileiro de Informática na Educação, p. 272. Disponível em: <http://br- ie.org/pub/index.php/wcbie/article/view/3226>. Acesso em: 15 jun. 2018.

Neto, W. C. B. and Lorenzini, I. P. (2017) "Sistema Tutor Inteligente para Auxílio na Alfabetização de Crianças Surdas em um Contexto Bilíngüe", In: XXIII Workshop de Informática na Escola p. 10 . Disponível em: <http://www.brie.org/pub/index.php/wie/article/view/2162/1928>. Acesso em: 15 jun. 2018.

Pereira, A., Kniphoff, M. E. J. and Frozza, R. (2007) "Ambiente Virtual de Aprendizagem Apoiado por um Agente Pedagógico de Acompanhamento para Alunos PNEEs Surdos”, In: Workshop em Informática na Educação, p. 4. Disponível em: <http://www.br-ie.org/pub/index.php/sbie/article/viewFile/648/634>. Acesso em: 15 jun. 2018.

Reinoso, L. and Tavares, O. (2015) "MVLIBRAS: ambiente digital para comunidades de aprendizagem com recursos inclusivos para surdos", In: XXVI Simpósio Brasileiro de Informática na Educação, p. 772. Disponível em: <http://brie.org/pub/index.php/sbie/article/view/5358>. Acesso em: 15 jun. 2018.

Rocha, D. F. S., Santana Pinto, I. B. and Silva, R. A. (2015) "AssistLIBRAS: Uma Ferramenta de Autoria para a Construção de Sinais da LIBRAS”, In: Revista Brasileira de Informática na Educação, v. 23, n. 02, p. 190.

Rocha, P. R., Lima, R., Macedo, R. et al. (2016) "Gamificação: Um aplicativo para o ensino da Língua Brasileira de Sinais", In: V Congresso Brasileiro de Informática na Educação, p. 896 . Disponível em: <http://brie.org/pub/index.php/wcbie/article/view/7014>. Acesso em: 15 jun. 2018.

Rodrigues, P. R. and Alves, L. R. G. (2014) "Criar e compartilhar games: novas possibilidades de letramento digital para crianças surdas.", In: CINTED- Novas Tecnologias na Educação, v. 12, n. 2.

Rodrigues, Y. W S., Silva, N. G. R., Silva, E. V. et al. (2017) "SAEPS: Uma ferramenta de apoio à alfabetização do surdo", In: XXVIII Simpósio Brasileiro de Informática na Educação, p. 4.

Mendonça, A. F. (2009) "Docência online: comunicação mediada por computadores em rede na prática docente", In: XV CIAED - Congresso Internacional Abed de E d u c a ç ã o a D i s t ân c i a.Disponível e m: $<$ http://www.abed.org.br/congresso2009/CD/trabalhos/552009101310.pdf>. Acesso em: 15 jun. 2018.

Santarosa, L. M. C., Passerino, L., Basso, L. O., et al. (2007) "Acessibilidade em Ambientes de Aprendizagem por Projetos 1: construção de espaços virtuais para inclusão digital e social de PNEEs", In: CINTED- Novas Tecnologias na Educação, v. 5, n. 1 .

Santarosa, L. M. C. and Silva, A. A. (2007) "Aquisição da Escrita de Sinais por Crianças Surdas através de Ambientes Digitais”, In: CINTED- Novas Tecnologias na Educação, v. 5, n. 2.

Santos, C. P. and Loose, C. (2017) "Estratégias Tecnológicas de Interação e Mediação para o Ensino de Geometria Espacial: Um Estudo de Caso com Alunos S u r d o s", I n : XXIII Workshop de Informática na Escola, p. 11. Disponível em: < 
VII Congresso Brasileiro de Informática na Educação (CBIE 2018)

Anais do XXIV Workshop de Informática na Escola (WIE 2018)

http://www.br-ie.org/pub/index.php/wie/article/view/7218>. Acesso em: 15 jun. 2018.

Santos, M. A. R. and Favero, E. L. (2014) "MCHQ-Alfa: Uma Proposta de Ferramenta para Aprendizagem da Língua Portuguesa na Educação de Surdos Utilizando o Potencial das Histórias em Quadrinhos Mediada por Mapa Conceitual”, In: Novas Tecnologias na Educação, v. 12, n. 1.

Santos, L. C. M., Miranda, T., Icó, M. A., et al. (2014) "Um jogo para aprender LIBRAS e português nas séries iniciais utilizando a tecnologia da realidade a u m e $\mathrm{n}$ t a d a", In: XXVIII Simpósio Brasileiro de Informática na Educação, p.1118. Disponível em: <http://br-ie.org/pub/index.php/sbie/article/view/3057>. Acesso em: 15 jun. 2018.

Santos, R. E. S., Magalhães, C. V. C., Maciel, J., et al. (2014) "Informática na educação especial: uma discussão no contexto da educação de surdos", In: XXVIII Simpósio Brasileiro de Informática na Educação, p. 622. Disponível em: <http://brie.org/pub/index.php/sbie/article/view/2992>. Acesso em: 15 jun. 2018.

Sarinho, V. T. (2017) "LIBRASZap - Um Jogo Baseado em Mensagens Instantâneas para Avaliação de Conhecimentos na Língua Brasileira de Sinais", In: Revista Brasileira de Informática na Educação, v. 25, n. 01, p.44.

Sarmento, V., Santos, D. L., Barros, F., et al. (2017) "Pessoas aparecem sinalizando e fica algo bem próximo a realidade: o software Lepê como possibilidade para uso do trabalho docente na educação de surdos". In: XXIII Workshop de Informática na Escola, p.235. Disponível e m: <ht p://www.brie.org/pub/index.php/wie/article/view/7241>. Acesso em: 15 jun. 2018.

Soares, M. I. S., Júnior, C. G. F., Silva, L. C., et al. (2014) "VISUAL JO2: Um Objeto de Aprendizagem para o Ensino de Programação Java a Deficientes Físicos e Auditivos através do Estímulo Visual - Um Estudo de Caso", In: CINTED-Novas Tecnologias na Educação, v. 12, n. 2.

Souza, V. C. and Pinto, S. C. C. S. (2007) "Customizando ambientes na web para a língua brasileira de sinais usando web-services", In: Revista Brasileira de Informática na Educação, v. 15, n. 2.

Tavares, O., Reinoso, L. and Almeida, W. (2017) "CAP-APL: plataforma para criação e uso de arquiteturas pedagógicas para aprendizagem de Português e LIBRAS" In: XXIII Workshop de Informática na Escola, p. 466. Disponível em: <http://www.brie.org/pub/index.php/sbie/article/view/7575>. Acesso em: 15 jun. 2018.

Toschi, M. S. (2010) "Leitura na tela: da mesmice à inovação", In: Goiânia: PUC Goiás.

Valente, J. A. (1999) “O computador na sociedade do conhecimento.” In: Campinas, SP: Unicamp/NIED, 1999.

Vieira, M. C., Corrêa, Y., Cheiran, J. F. P., et al. (2014) "Contribuições da Teoria da Aprendizagem Multimídia e da Usabilidade para aprendizagem de LIBRAS e Língua Portuguesa por meio de aplicativos móveis.", In: CINTED- Novas Tecnologias na Educação, v. 12, n. 2. 